\title{
CO-MORBIDITIES AMONG EPILEPSY PATIENTS: EXPERIENCE IN BANGLADESH
}

\author{
RAJIB NAYAN CHOWDHURY ${ }^{1}$, ATM HASIBUL HASAN ${ }^{2}$, KAZI MOHIBUR RAHMAN ${ }^{3}$, SUDIP RANJAN DEB ${ }^{4}$
}

\begin{abstract}
:
Objective: To determine comprehensively all the major comorbid diseases observed among epilepsy patients.

Methods: In this observational study, 1168 patients were recruited from outpatient based epilepsy clinic in a tertiary care hospital. Four categories of comorbid conditions namely neuropsychiatric, developmental (mental retardation, cerebral palsy), pain disorder (migraine) and others (hypertension, diabetes, stroke) were evaluated in these patients through a prefilled questionnaire and data were then analyzed. Epilepsy were broadly classified into generalized epilepsy (GE), localization related epilepsy (LRE), symptomatic and unclassified.
\end{abstract}

\begin{abstract}
Result: Among the 1168 subjects we included in this study, 71.5\% were male. The most common age group at the time of interview was 11-20 years (36.8\%). Only 29 (2.5\%) respondents were older than 60 years. Among the listed comorbid conditions, mental retardation was the most common entity (15.5\%), followed by psychiatric disorder (12.8\%), hypertension (5.6\%), migraine (5.4\%) and cerebral palsy (5.0\%). Only 1.5\% had diabetes and $0.6 \%$ had stroke. Mental retardation and cerebral palsy were more common and significantly associated ( $p=0.0001$ and 0.005$)$ with GE patients (95 and 44), psychiatric disorder was also common among GE patients (108)) with a $p$ value of 0.0001. But migraine was more common and significantly associated ( $p=0.0001$ ) with LRE patients (46). Stroke was only present in symptomatic epilepsy group (7) and diabetes was present only in GE patients (19). Both were significant ( $p=0.0001$ and 0.01). But hypertension among different epilepsy groups was not significant $(p=0.08)$.
\end{abstract}

Conclusion: Neuropsychiatric, developmental and pain disorder are common comorbid associations within different epilepsy syndromes which may need special care during management of epilepsy patients.

Key Words: Comorbidity, Hypertension (HTN), Diabetes Mellitus (DM)

\section{Introduction:}

An estimated 59 million people is affected by epilepsy worldwide $^{1}$. The prevalence of active epilepsy, range from $0.2-4.1 \%^{2}$. Although defined by presence of recurrent seizure, epilepsy has different dimensions including abnormality in cognition, psychiatric status, social adaptation and many more. In the past centuries people with epilepsy were frequently segregated in colonies ${ }^{3}$. Epileptologists subsequently sought to provide a more representative picture of the consequences of epilepsy in people who lived in the community 4 . The term "comorbidity" refers to one or more other disease among people with an index disease ${ }^{5}$. It is often difficult to determine whether a clinical entity should be marked as comorbidity or complication of the disease itself. The working definition of complication is regarded as existence of a second disease when the occurrence of an index disease is required ${ }^{6}$. Few years back, at the National Institutes of Health conference Curing Epilepsy 2007: Translating Discoveries into Therapies, the prevention and reversal of the comorbidities of epilepsy were identified as a major new benchmark area for research. A number of conditions have been reported to be comorbid with epilepsy, including psychiatric disorders and certain pain disorders, the improved knowledge base of which is very much important. Firstly, the awareness of comorbidities can improve diagnosis. Secondly, some comorbidities may influence the prognosis of epilepsy, as has been shown for migraine ${ }^{7}$. Thirdly, the recognition of comorbidities can inform therapeutic choices. It can either create opportunities to treat two conditions with a single drug or impose therapeutic limitations. Thus the knowledge of comorbidities may provide insight into pathogenesis by revealing shared neurobiologic

1. Assistant Professor of Neurology, Dhaka Medical College Hospital

2. Medical Officer, OPD (Medicine), Dhaka Medical College Hospital

3. Assistant Professor of Neurology, Dhaka Medical College Hospital

4. Resident Physician, Dhaka Medical College Hospital

Bangladesh J Medicine 2013; 24 : 65-69 
mechanisms underlying multiple disorders. In this study we tried to find out the distribution of different comorbid condition in different types of epilepsy patients. The list of comorbidities were selected on the basis of prior research ${ }^{8-10}$.

\section{Materials and Methods:}

This cross sectional study was carried out in specialized epilepsy weekly outpatient clinic of Dhaka medical college from January 2008 to July 2011 which included 1168 epilepsy patients. Epilepsy syndromes were broadly classified into four categories using the classification system of International League Against Epilepsy (ILAE) namely, generalized epilepsy (GE), localization related epilepsy (LRE), symptomatic and unclassified. Each patient was evaluated and classified by consultant neurologist. Four categories of comorbid conditions namely neuropsychiatric, developmental (mental retardation, cerebral palsy), pain disorder (migraine) and others (hypertension, diabetes, stroke) were evaluated in these patients through a prefilled questionnaire. These comorbidities were selected on the basis of our knowledge base from prior researches. Statistical analysis of their response was done with the help of SPSS version 16.0.

\section{Result:}

Among the 1168 subjects we included in this study, $71.5 \%$ were male. The most common age group at the time of interview was $11-20$ years $(36.8 \%)$, followed by $27.7 \%$ young adults who were at $21-30$ years of age (Table-I). Only 29 (2.5\%) respondents were older than 60 years. Among the listed comorbid conditions, mental retardation was the most common entity (15.5\%), followed by psychiatric disorder (12.8\%), hypertension (5.6\%), migraine $(5.4 \%)$ and cerebral palsy $(5.0 \%)$. Only $1.5 \%$ had diabetes and $0.6 \%$ had stroke (Figure-I). Further subgroup analysis of developmental disorder showed that mental retardation and cerebral palsy were more common and significantly associated ( $p=0.0001$ and 0.005$)$ with GE patients (95 and 44) than LRE (75 and 17) respectively (Table-II). Similarly psychiatric disorder was also common among GE patients (108) than LRE (50) with a $p$ value of 0.0001 . But migraine was more common and significantly associated $(p=0.0001)$ with LRE patients (46) than GE (20) (Table-II). Stroke was only present in symptomatic epilepsy group (7) and diabetes was present only in GE patients (19). Both were significant with $p$ value of 0.0001 and 0.01 respectively. Though hypertension was common, the difference for hypertension among different epilepsy groups was not significant $(p=0.08)$.
Table I

Socio demographic profile of the patients $(N=1168)$

\begin{tabular}{llcc}
\hline Parameter & & $\mathrm{n}$ & $\%$ \\
\hline Age & 0-10 yrs & 217 & 18.6 \\
& 11-20 yrs & 430 & 36.8 \\
& 21-30 yrs & 323 & 27.7 \\
& 31-40 yrs & 109 & 9.3 \\
& 41-50 yrs & 31 & 2.7 \\
& $>60$ yrs & 29 & 2.5 \\
& Male & 835 & 71.5 \\
& Female & 333 & 28.5 \\
\hline
\end{tabular}

Table II

Common comorbid conditions in different class of epilepsy

\begin{tabular}{lccccc}
\hline Parameter & \multicolumn{5}{c}{ Epilepsy class } \\
& GE & LRE & $\begin{array}{c}\text { Sympto- } \\
\text { matic }\end{array}$ & $\begin{array}{c}\text { Unclass- } \\
\text { ified }\end{array}$ & $\begin{array}{c}p \\
\text { value }\end{array}$ \\
\hline Mental & 95 & 75 & 14 & 7 & 0.0001 \\
retardation & & & & & \\
Cerebral Palsy & 44 & 17 & 0 & 0 & 0.005 \\
Psychiatric & 108 & 50 & 0 & 0 & 0.0001 \\
disorder & & & & & \\
Migraine & 20 & 46 & 0 & 0 & 0.0001 \\
Stroke & 0 & 0 & 7 & 0 & 0.0001 \\
Hypertension & 46 & 16 & 7 & 0 & 0.08 \\
Diabetes & 19 & 0 & 0 & 0 & 0.01 \\
\hline
\end{tabular}

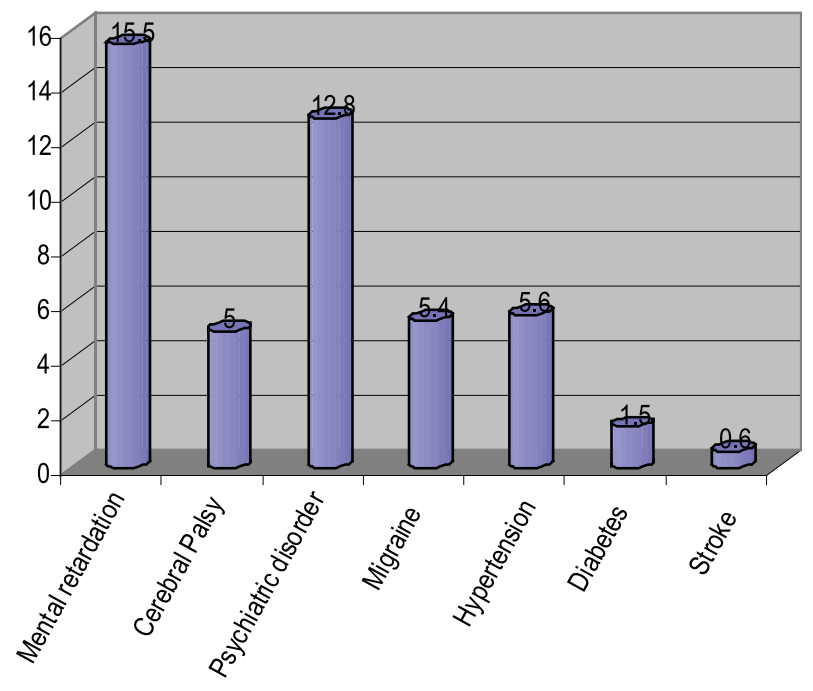

Fig.-1 


\section{Discussion:}

Researchers have reported that $4 \%$ to $10 \%$ of all children experience a seizure at some time in their lives. By age 20, however, only $1 \%$ of the population has a diagnosis of seizures or epilepsy ${ }^{11}$. Many patients with developmental disorders present with an epilepsy comorbidity. For example, seizures appear in more than $20 \%$ of children with cerebral palsy, unspecified cognitive impairment, pervasive developmental delay, as well as in specific genetic syndromes associated with cognitive impairment. In addition, there are different types of epilepsy that may be associated with different types of developmental disability. Both the paper electroencephalogram (EEG) and the video EEG remain the most valuable tools that are used to evaluate patients with both epilepsy and a developmental disorder ${ }^{12}$. Investigators have studied the co-morbidity of epilepsy and numerous childhood onset conditions, including cerebral palsy, autism, Down syndrome, and mental retardation ${ }^{13-15}$. Recent reports about children with cerebral palsy indicate that 35\% have a history of epilepsy, with the highest prevalence in children with spastic hemiplegia (66\%), followed by those with quadriplegia (43\%), and diplegia $(16 \%)^{16}$. Similar to the reports from other investigators, mental retardation and cerebral palsy were significantly associated with GE patients ( $\mathrm{p}$ value 0.0001 and 0.005 respectively) in our study. The relationship between seizure disorders and mental retardation is a complex one. When considered across the spectrum of mental retardation, the frequency and severity of epilepsy varies inversely with the level of mental retardation ${ }^{17}$. Among individuals with mild retardation, the distribution of various subtypes of seizures approximates the general population. This observation probably relates to lower rates of neuroanatomical abnormalities or metabolic disorders. The clinical response to anticonvulsant is also comparable to the general population. Among individuals with severe/profound retardation, increased rates of underlying neurological abnormalities are manifested by a higher frequency of mixed seizure disorders, and an onset of seizures in infancy or early childhood ${ }^{17,18}$. This factor contributes to higher rates of uncontrollable seizures and neuropsychiatric complications. These clients may also require multiple anticonvulsants (AEDs), increasing the risk for medication side effects and pharmacokinetics interactions ${ }^{19}$. Even with AED combinations, many clients continue to experience frequent seizures and risk further cognitive or behavioral deterioration. The degree of clinical deterioration relates to frequent seizures; early age of onset, level of seizure control with the time required, and the presence of underlying neuroanatomical lesions ${ }^{20,21}$. There is also a considerable overlap between mental retardation and other developmental disorders with psychiatric diseases. Population studies indicate that rates of psychiatric disorders approach $40 \%$ of the population of individuals with mental retardation ${ }^{22}$. The categories of psychopathology vary depending on the level of mental retardation and deficits in adaptive behavior, and genetic vulnerability to disorders such as affective illness, anxiety disorders, and schizophrenia ${ }^{23}$.

In our study for the purpose of simplification, we grouped disorders like depression, anxiety, panic attacks, schizophrenia all together under the heading psychiatric disorder. About $12.8 \%$ of our patients had at least one variety of this psychiatric diagnosis along with epilepsy. The significant association was with GE patients $(p=0.0001)$ Psychiatric disorders have been a longstanding concern in tertiary care epilepsy centres. Recent population-based studies that use contemporary diagnostic criteria, such as the Diagnostic and Statistical Manual of Mental Disorders IV (DSM-IV) and the International Classification of Diseases, are crucial to define the extent of the problem. Tellez- Zenteno and co-workers ${ }^{24}$ investigated mental health status in 36984 people who participated in the Canadian Community Health Survey $1 \cdot 2$. The World Mental Health Composite International Diagnostic Interview was used to derive 12 -month and lifetime rates of $D S M-I V$ axis I disorders. Patients with epilepsy were more likely to report lifetime anxiety disorders and suicidal thoughts, and lifetime major depression was significantly higher after adjustment for covariates. Population-based investigations of the associations between epilepsy and psychiatric disorders compared with other chronic health conditions are important. Recent studies of targeted psychiatric symptoms in large, community-based populations investigate this question. For example, Ettinger and co-workers ${ }^{25,26}$ studied symptoms of depression and bipolar disorder with self report inventories. Bipolar symptoms were significantly elevated in patients with epilepsy $(12 \cdot 2 \%)$ compared with patients with migraine $(7 \cdot 2 \%)$, asthma $(5 \cdot 9 \%)$, diabetes $(3 \cdot 2 \%)$, or controls $(1 \cdot 7 \%)$. Many studies have consistently shown an increased risk of depression after epilepsy onset, some data also shows that the depressed individuals have increased risk of developing epilepsy. This bidirectional relationship supports the hypothesis of overlapping mechanisms for the two disorders ${ }^{27-32}$. Whether the population based prevalence rates of psychiatric 
disorders are increased in patients with epilepsy compared with people with other neurological disorders (eg, multiple sclerosis) needs to be determined and is a crucial question with regard to the specificity of association. Findings also suggest that psychiatric, cognitive and behavioral problems might even antedate the diagnosis of epilepsy ${ }^{33,34}$.

Migraine and epilepsy are associated independent of seizure type, etiology, age at onset, or family history of epilepsy ${ }^{35}$. These two conditions might share a common genetic susceptibility ${ }^{36}$ or a common environmental risk factor, such as traumatic head injury $^{37}$. Depression and migraine with aura act synergistically in increasing the risk of incident unprovoked seizure, suggesting that these three disorders may result from a shared underlying mechanism ${ }^{38}$. A recent study reported an increased frequency of migraine in the relatives of individuals with benign rolandic epilepsy, providing evidence for a shared genetic susceptibility to migraine and some forms of epilepsy ${ }^{36}$. Finding in our study was a little bit different. Migraine was strongly associated with LRE $(p=0.0001)$. There are several possible explanations for the co-occurrence of these two disorders ${ }^{39-41}$. First, since migraine and epilepsy are both relatively common, they may co-occur frequently by chance. Second, they may be mistaken for one another, as migraine and epilepsy-particularly partial seizures - have considerable overlap of clinical symptoms. Third, one may cause the other-migraine may trigger or evolve into a seizure ("migralepsy") 41 and seizures may cause post-ictal headache. Fourth, they may have a shared pathophysiology, such as ion channel dysfunction, that is not necessarily the result of shared genetic factors. Finally, migraine and epilepsy may have a shared genetic cause, a topic addressed in this issue of Neurology by Deprez et al., in their description of linkage of familial occipitotemporal lobe epilepsy and migraine with visual aura to chromosome $9 q^{42}$. The risk of migraine has been found to be more than twice as high in individuals with epilepsy as in those without epilepsy ${ }^{35}$.

Stroke was only associated with symptomatic epilepsy group ( $p=0.00001)$. Engel ${ }^{43}$ also reported similarly that symptomatic epilepsy results from known condition such as neoplasm, vascular malformation, stroke, scar etc. High blood pressure was also reported by a good fraction of epilepsy patients. It is a risk factor for stroke, which itself is a risk factor for epilepsy, but a direct association between high blood pressure and epilepsy was not found in our study and not reported in any literature yet. But complying with the reports of other studies the association of diabetes was significant ( $p=0.01)$ There is evidence to suggest that antibodies to glutamic acid decarboxylase, an auto-antigen implicated in type 1 diabetes, are also associated with the development of epilepsy ${ }^{44}$.

\section{Conclusion:}

The neuropsychiatric, developmental and pain disorders are quite common comorbid conditions among epilepsy patients. Similarity in pathophysiologic mechanisms might cause these overlapping conditions within same individual. Treating physicians should be attentive to the possibility of these comorbidities in patients with epilepsy. We are in dare need of future research to examine the impact of comorbidities on treatment outcomes in epilepsy.

\section{Conflict of interest: None}

\section{References:}

1. World Health Organisation. Epilepsy: etiology, epidemiology and prognosis. Fact sheet 165. Geneva: WHO, 2001.

2. Banerjee PN, Filippi D, Hauser WA. The descriptive epidemiology of epilepsy-a review. Epilepsy Res 2009; 85:31-45.

3. Fox JT. The response of epileptic children to mental and educational tests. British Journal of Medical Psychology 1924; 4: 235-248.

4. Lennox WG. Epilepsy and related disorders. Boston: Little, Brown, and Co, 1960.

5. Feinstein AR. The pre-therapeutic classification of co-morbidity in chronic disease. J Chron Dis 1970; 23:455-69.

6. Schellevis FG. Comorbidity-definitions and methodological aspects. In : Chronic disease in general practice. Comorbidity and quality of care. (thesis). Nijmegen: Catholic University Nijmegen:1993.

7. Velioglu SK, Boz C, Ozmenoglu M. The impact of migraine on epilepsy: a prospective prognosis study. Cephalalgia 2005; 25:528- 535 .

8. Boro A, Haut S. Medical comorbidities in the treatment of epilepsy. Epilepsy Behav 2003; 4(supp1 2):S2-S12.

9. Gilliam FG, Mendiratta A, Pack AM, Bazil CW. Epilepsy and common comorbidities: improving the outpatient epilepsy encounter. Epileptic Disord 2005; 7(suppl 1):S27-S33.

10. Swinkels WA, Kuyk J, van Dyck R, Spinhoven P. Psychiatric comorbidity in epilepsy. Epilepsy Behav 2005; 7:37-50.

11. Hauser, W. The prevalence and incidence of convulsive disorders in children. Epilepsia,1994; 35(Suppl. 2), S1-6. 
12. Persad V, Thompson MD, Percy ME. Epilepsy and developmental disability. Journal on Developmental Disabilities; 10 (2): 123-152.

13. Brodtkrob, E. The diversity of epilepsy in adults with severe developmental disabilities: Age at seizure onset and other prognostic factors. Seizure 1994; 3, 277-285.

14. Johannsen, P., \& Christensen, J. Epilepsy in Down syndrome Prevalence in three groups. Seizure 1996; $5,121-125$.

15. Volkmar, F., \& Nelson, D. S. Seizure disorders in autism. Journal of the American Academy of Child and Adolescent Psychiatry1990; 29, 127- 129.

16. Singhi, P., Jagirdar, S., Khandelwal, N., \& Malhi, P. Epilepsy in children with cerebral palsy. Journal of Child Neurology 2003; 18, 174-179.

17. Roberts JKA. "Neuropsychiatric Complications of Mental Retardation" In Stavarki, C (Ed) Psychiatric Clinics of North America 9(1). WB Saunders Phila. 1986; p647-655.

18. Ratey, JJ and Dynek, MP. "Neuropsychiatry of Mental Retardation and Cerebral Palsy" in Fogel, BS and Schiffer, RB (Eds) Neuropsychiatry, Williams and Wilkins Baltimore. 1996; P549-70.

19. Reynolds, EH. " Antiepileptic Drugs and Psychopathology" In Trimble, MR (Ed) The Psychopharmacology of Epilepsy. John A Wiley New York. 1985; p49-65.

20. Dodrill, CB. "Neuropsychological Aspects of Epilepsy" in Biller, BJ and Kathol, RG. Psychiatric Clinics 15(2) WB Saunders, Phila p 383-93.

21. Trimble, MR; Ring HA; and Schmitz, B. “ Neuropsychiatric Aspects of Epilepsy" in Fogel MS and Schiffer, RB (Des) Neuropsychiatry Williams and Wilkins, Baltimore 1996; p 771-804.

22. Parsons, JA; May JG; and Menolascino, FJ. "The Nature and Incidence of Mental Illness in Mentally Retarded Individuals" in Menolascino, FJ and Stark, $\mathrm{J}$ (Des) Handbook of Mental illness in the Mentally Retarded. New York Plenum 1984.

23. Ruedrich, S and Menolascino, FJ. "Dual Diagnosis of Mental retardation and Mental Illness" in Menolascino, FJ and Stark, J (Des) Handbook of Mental Illness in the Mentally Retarded Plenum. New York 1984; p 45-82.

24. Tellez-Zenteno JF, Patten SB, Jette N, Williams J, Wiebe S. Psychiatric comorbidity in epilepsy: a population-based analysis. Epilepsia 2007; 48: 2336-44.

25. Ettinger A, Reed M, Cramer J. Depression and comorbidity in community-based patients with epilepsy or asthma. Neurology 2004; 63: 1008-14.

26. Ettinger AB, Reed ML, Goldberg JF, Hirschfeld RM. Prevalence of bipolar symptoms in epilepsy vs other chronic health disorders. Neurology 2005; 65: 53540 .

27. Edeh J, Toone B. Relationship between interictal psychopathology and the type of epilepsy. Results of a survey in general practice. $\mathrm{Br} \mathrm{J}$ Psychiatry 1987;151:95-101.
28. Jacoby A, Baker GA, Steen N, Potts P, Chadwick DW. The clinical course of epilepsy and its psychosocial correlates: findings from a U.K. Community study. Epilepsia 1996;37:148-161.

29. Forsgren L, Nystrom L. An incident case-referent study of epileptic seizures in adults. Epilepsy Res 1990; 6:66-81.

30. Hesdorffer DC, Hauser WA, Annegers JF, Cascino G. Major depression is a risk factor for seizures in older adults. Ann Neurol 2000; 47:246-249.

31. Kanner AM. Epilepsy, suicidal behaviour, and depression: do they share common pathogenic mechanisms? Lancet Neurol 2006; 5:107- 108.

32. LaFrance WC Jr, Kanner AM, Hermann B. Psychiatric comorbidities in epilepsy. Int Rev Neurobiol 2008; 83:347-383.

33. Austin JK, Harezlak J, Dunn DW, Huster GA, Rose DF, Ambrosius WT. Behavior problems in children before fi rst recognized seizures. Pediatrics 2001; 107: 115-22.

34. Dunn DW, Harezlak J, Ambrosius WT, Austin JK, Hale B. Teacher assessment of behaviour in children with new-onset seizures. Seizure 2002; 11: 169-75.

35. Ottman R, Lipton RB. Comorbidity of migraine and epilepsy. Neurology 1994; 44:2105-2110.

36. Clarke T, Baskurt Z, Strug LJ, Pal DK. Evidence of shared genetic risk factors for migraine and rolandic epilepsy. Epilepsia 2009; 50:2428- 2433.

37. Gaitatzis A, Carroll K, Majeed A, Sander JW. The epidemiology of the comorbidity of epilepsy in the general population. Epilepsia 2004; 45:1613-1622.

38. Hesdorffer DC, Ludvigsson P, Hauser WA, Olafsson $\mathrm{E}$, Kjartansson O. Co-occurrence of major depression or suicide attempt with migraine with aura and risk for unprovoked seizure. Epilepsy Res 2007; 75:220223.

39. Andermann E, Andermann F. Migraine-epilepsy relationships: epidemiological and genetic aspects. In: F A, E L, eds. Migraine and epilepsy. Boston: Butterworths, 1987.

40. Bigal ME, Lipton RB, Cohen J, Silberstein SD. Epilepsy and migraine. Epilepsy Behav 2003;4 suppl 2:S13-24.

41. Milligan TA, Bromfield E. A case of "migralepsy." Epilepsia 2005;46 suppl 10:2-6.

42. Deprez L, Peeters K, Van Paesschen W, et al. Familial occipitotemporal lobe epilepsy and migraine with visual aura: linkage to chromosome 9q. Neurology 2007;68: 1995-2002.

43. Engel, J. Research on the human brain in an epilepsy surgery setting. Epilepsy Research 1998; 32(1-2), 1-11.

44. Nesbitt V, Prudhoe S, Kumar R, Cheetham T. Is there an association between type 1 diabetes and epilepsy in childhood? Archives of Disease in Childhood 2010;95:A70. 